\title{
Establecimiento de Normas de Aula para Favorecer la Voz del Alumnado. Estudio de Caso Español
}

\author{
Establishing the Classroom Rules to Encourage Student Voice. \\ Spanish Case Study
}

\author{
Marta Terrén* \\ Auxiliadora Sales \\ Universitat Jaume I
}

\begin{abstract}
Este artículo describe el proceso de investigación-acción desarrollado en dos aulas de Educación Primaria de un centro en transformación hacia el modelo de escuela intercultural inclusiva. Partimos del análisis del nivel de participación del alumnado, la mirada que otorga el profesorado a su práctica docente y las metodologías participativas implantadas, con la finalidad de indagar acerca del papel que se otorga a la voz del alumnado y consensuar el establecimiento de una estrategia que favorezca su protagonismo y participación, centrada en la reelaboración de las normas del aula por parte del alumnado. Los resultados señalan que a pesar de las diferencias existentes en ambas aulas, la aplicación de la estrategia es posible. Podemos concluir que, poner en práctica estrategias participativas permite la toma de conciencia de sus beneficios, ampliando el interés al respecto y facilitando una reflexión crítica desde una perspectiva intercultural inclusiva.
\end{abstract}

Descriptores: Voz del alumnado, Escuela intercultural inclusiva, Investigaciónacción, Normas de aula.

\begin{abstract}
This article describes an action-investigation process developed in two elementary level classrooms of a school in a transformation process to become an intercultural inclusive school. We start with an analysis of the participation level of the students, teachers teaching styles and participating methodologies that are used, in order to inquire about the role that the student's voice and to reach an agreement to establish a new strategy, which favors its role and participation and that is centered in the re-elaboration of classroom rules by the students. The results indicate that despite differences in both classrooms, the implementation of the strategy is plausible. We can conclude that, implementing participatory strategies allows the realization of its benefits, extending the interest thereon and facilitating critical reflection from an inclusive intercultural perspective.
\end{abstract}

Keywords: Student voice, Intercultural inclusive school, Action-research, Classroom rules. 


\section{Introducción}

El modelo de escuela intercultural inclusiva apuesta por dar respuesta a la diversidad de necesidades que presenta el alumnado y por el incremento de su participación, reduciendo la exclusión (Moliner y St-Vicent, 2014). Aboga por una educación para todos, en igualdad de condiciones, y por la transformación educativa hacia valores democráticos de igualdad, respeto, autonomía y solidaridad. Este modelo se encuentra inmerso en un proceso de transformación cultural y social, que busca el establecimiento de un diálogo intercultural y avanzar hacia el aprendizaje dialógico, centrado en la construcción de significados compartidos, al considerar al alumnado capaz de construir su propio conocimiento, de forma crítica y reflexiva (Sales, Moliner y Ruiz, 2013).

La consecución de una escuela en la que se contemple el modelo intercultural inclusivo, lleva implícito un proceso de construcción democrática, que requiere la participación plena de toda la comunidad en la construcción del conocimiento colectivo y el compromiso ciudadano e investigador para el cambio social (Sales, 2011). Impulsar dicha participación, a la que cabe añadir el derecho de todos a tomar decisiones que se conviertan en acciones (Ramos, 2013), permitirá un incremento a medio y largo plazo de la implicación familiar, docente y no docente, y como aspecto a destacar, del propio alumnado (Montalbán, 2006). Desde esta perspectiva, se considera al alumnado como agente capaz de participar plenamente en el hecho educativo, reconociendo su papel como actor principal y destinatario de la gestión y la vida del aula (Traver, Sales, Moliner, 2010).

Puesto que a participar se aprende participando, es necesario otorgar al alumnado responsabilidades y compromisos graduales que lo encaminen hacia el ejercicio de la participación (Martínez, 2006). En este sentido, remitimos al término Voz del Alumnado, todavía poco común en nuestro país, a pesar de que la idea fundamental viene siendo desarrollada bajo otros epígrafes teóricos, como la inclusión educativa, la escuela democrática, los derechos de la infancia, etc. Éste se refiere a la iniciativa de aumentar el protagonismo del alumnado en la toma de decisiones, logrando una participación auténtica que se convierta en un objetivo pedagógico relevante, otorgándole una posición privilegiada para alimentar cambios en la cultura escolar (Susinos, 2012). De tal modo, cualquier intento de mejora escolar será incompleta si se deja de lado dicha voz, puesto que el alumnado también posee los conocimientos y la posición apropiada para dar forma a aspectos educativos básicos (Rojas, Haya y Lázaro-Visa, 2012), a los que comúnmente no prestamos atención, pero podemos reconocer como importantes al considerar a los alumnos "testigos expertos" de la tarea del cambio (Rudduck y Flutter, 2007). Todo ello requiere de la presencia de profesorado capaz de analizar sus propias prácticas con una mirada renovada, que le permita replantearse sus rutinas de gestión del aula y el centro, y considerar necesaria la participación del alumnado en diferentes ámbitos de la vida escolar, ayudándole a pasar del silencio a la influencia y de la invisibilidad a la visibilidad (Rudduck y Flutter, 2007; Susinos y Ceballos, 2012; Susinos, Rojas y Lázaro, 2011).

Diversos autores hacen referencia a la existencia de un continuo de etapas o niveles de protagonismo, descritas por Nieto y Portela (2008) y Susinos y Ceballos (2012). El primer nivel se caracteriza por la unión entre el aprendizaje intergeneracional y la comunidad democrática, en sentido político, denominado por Fielding (2011) “comunidad democrática” (p. 38). Este nivel es entendido como un marco de relaciones 
que permite reflexionar sobre aspectos que preocupan e influyen a los alumnos, considerándolos "agentes activos que trabajan y aprenden conjuntamente con otros miembros de la comunidad escolar y que asumen compromisos que persiguen el bien común” (Rojas, Haya y Lázaro-Visa, 2012, p. 83). En el segundo nivel: alumnado investigador, los estudiantes adquieren protagonismo en el diseño y desarrollo de proyectos e intervienen en la mejora escolar, por medio de la retroalimentación, discusión de resultados y un papel activo en la toma de decisiones. El tercer nivel: alumnado como participante activo, considera válida la opinión del alumnado y permite su intervención en la toma de decisiones, aunque en menor frecuencia que los niveles anteriores. El cuarto nivel: escucha del alumnado, se caracteriza por convertirlo en informante y mera fuente de datos. El quinto y último nivel, denominado ausencia de consulta, consiste en la falta total de participación o consideración de la opinión del alumnado.

Avanzar entren los niveles descritos supone la puesta en práctica de estrategias educativas, que permitan entender la Voz del Alumnado como provisional, en desarrollo y múltiple. Centrándonos en las experiencias analizadas (Fielding, 2011; Susinos y Rodríguez-Hoyos, 2011), las estrategias comúnmente empleadas para favorecer la expresión y escucha del alumnado son: entrevistas, asambleas, encuestas, reuniones regulares de la plantilla y el alumnado, buzones de sugerencias, análisis de fotografías y dibujos, etc. A nuestro parecer, una de las propuestas más interesantes se centra en la implicación del alumnado en la elaboración y seguimiento de las normas de aula, como propone San Fabián (2008), para equilibrar la responsabilidad y la autonomía. Consiste en un proceso llevado a cabo por medio del consenso y negociación de las normas, que deben plantearse de forma sencilla, concreta y clara, comenzando con el establecimiento de unas pocas a las que dedicar un periodo de tiempo medio o largo, para facilitar su asimilación (Pérez, 1995). En este sentido, se entienden las normas como reglas fruto del consenso social, cumplidas voluntariamente por considerarlas válidas y necesarias para la convivencia del grupo (Pérez, 2011). Cuando el alumnado construye y se compromete con las normas de aula, se favorece su cumplimiento y satisfacción (Lorenzo, 2004), al sentirse protagonista del modelo establecido y alejarse del modelo impositivo y autoritario que, en contraposición, no facilita el mantenimiento de pautas de conducta constantes, independientemente del contexto (Pérez, 2007). La discusión, elaboración y votación, constituye un procedimiento de participación real, que convierte las normas en un contrato de vida común y permite que su palabra sea oída en igualdad de condiciones a la del adulto, favoreciendo la construcción de la ciudadanía democrática, el ejercicio de los derechos y el respeto de los deberes (Le Gal, 2005).

La presente revisión teórica se convierte en el punto de referencia y partida de la investigación, en la que se pretende identificar los niveles y estrategias de participación presentes en las aulas de un centro educativo concreto, en proceso de adquisición del modelo intercultural inclusivo. Con la finalidad de determinar y promover la puesta en práctica de estrategias que favorezcan la escucha y expresión de la voz del alumnado, situándolo en niveles elevados de participación, característica implícita al modelo de escuela intercultural inclusiva (Sales, 2011). 


\section{Diseño de la investigación}

\subsection{Contexto}

El proyecto de investigación se desarrolla durante los meses de mayo y junio de 2015 en un Centro Rural Agrupado de Educación Infantil y Primaria, constituido por dos aularios situados en la provincia de Valencia, España. Desde hace 7 años se encuentra inmerso en un proceso de transformación hacia una escuela intercultural inclusiva, de la mano del grupo de investigación Mejora Educativa y Ciudadanía Crítica, de la Universidad Jaume I (Castellón)․ En la actualidad se está desarrollando un proceso de Investigación Acción Participativa, que tiene como objetivo conocer con qué estrategias y bajo qué condiciones la participación democrática de la comunidad educativa contribuye a la construcción de una escuela intercultural inclusiva. El centro se caracteriza por su apertura al entorno y el protagonismo otorgado a las familias, mediante la gestión del Banco de Libros y la capacidad de decisión otorgada a la Asociación de Madres y Padres de Alumnos/as. Además han optado por la apertura del Consejo Escolar, mediante la creación de la Comisión Coordinadora, integrada por el Consejo y todas aquellas personas que deseen formar parte.

\subsection{Problema y objetivos de investigación}

Durante las diversas fases de investigación del proyecto mencionado, se realizan esfuerzos por contemplar la opinión del alumnado, aspecto difícil de conseguir debido a las dificultades que presenta el centro para gestionar y promover una verdadera escucha de su voz. Como corroborado el director, al afirmar que la voz del alumnado se pierde fácilmente, a pesar de tratar de favorecer su participación activa en la Comisión Coordinadora. Esto es debido a la falta de aplicación de dinámicas y estrategias desarrolladas de forma sistemática, cuya puesta en práctica se limita a momentos y temas puntuales, que derivan en una preparación forzada de la voz del alumnado. Así pues, resulta conveniente conocer el nivel de participación real del alumnado en el aula y el centro, para tratar de promover su mejora con estrategias educativas.

Partiendo del problema de investigación expuesto, las preguntas que pretendemos responder son: ¿qué niveles de participación se encuentran presentes en el aula?, ¿qué mirada otorga el tutor a la participación del alumnado en su práctica docente?, ¿qué estrategias y/o metodologías se ponen en práctica para favorecer la escucha de la voz del alumnado? y ¿qué estrategias establecer para potenciar dicha voz? Éstas serán respuestas para alcanzar el objetivo principal de la investigación, tratar de fomentar la expresión y escucha del alumnado mediante la identificación y establecimiento de estrategias que la favorezcan, concretado en cuatro objetivos específicos:

- Indagar sobre los niveles de participación del alumnado en el centro educativo y el aula.

- Identificar las estrategias empleadas para permitir la participación y tener en cuenta la voz el alumnado en el contexto del aula.

${ }^{1}$ Proyecto I+D: "Participación comunitaria y escuela democrática: estrategias para la formación de una ciudadanía crítica" (p. 11B2013-32). Convocatoria investigación Bancaja-UJI 2013 
- Compartir significados sobre la participación y favorecer la toma de decisiones.

- Poner en práctica y evaluar estrategias que fomenten la participación, la toma de decisiones y la escucha del alumnado dentro del aula.

\subsection{Metodología}

El estudio centra su atención en comprender una realidad educativa concreta, indagando en su espacio natural, con la intención de establecer acciones que favorezcan la mejora de la situación de partida. Con tal propósito se desarrolla un análisis y reflexión inicial, continuó y final, mediante el empleo de diversas técnicas específicas, que deriven en posibles propuestas aplicables al contexto real, estableciendo una relación directa con los sujetos implicados. El estudio se desarrolla mediante la metodología de investigaciónacción, empleada por el profesorado y la comunidad educativa, con la intención de reflexionar y establecer acciones sobre los problemas existentes en su práctica (Elliott, 2011). Ésta se convierte en un poderoso instrumento para cuestionar y reconstruir las prácticas sociales y educativas, con la intención de comprenderlas y explicarlas, mediante la vinculación del cambio y el conocimiento (Latorre, 2003). La investigación se desarrolla a través de una espiral de ciclos, durante los que se suceden fases de planificación, acción, observación y reflexión. Se parte de la identificación de un problema inicial, del que se recogen evidencias y analizan los datos obtenidos, a partir de los que desarrollar un plan de acción flexible, seguido de la acción y reflexión, derivando en la planificación de un nuevo ciclo (Elliott, 2011; Latorre, 2003).

\subsection{Participantes}

Los participantes de la investigación han adquirido el máximo protagonismo, al emplear un planteamiento y desarrollo abierto y flexible, mediante la construcción y modificación de los ciclos, atendiendo a sus necesidades e intereses. Para evitar limitar las fronteras de la investigación a un único contexto, es desarrollada en el interior de dos aulas, determinadas en base a los siguientes criterios: predisposición del tutor del aula, implicando a docentes con iniciativa, preocupados por su práctica educativa y dispuestos a promover mejoras; posesión y empleo de experiencias educativas y metodologías diversas, contando con variedad de prácticas y opiniones, y tomar como referencia un grupo-clase de cada aulario.

Tales criterios llevaron a determinar como principales protagonistas de la investigaciónacción un total de veinte alumnos, de edades comprendidas entre nueves y doce años, y dos tutores, pertenecientes a aulas diferentes, a las que pasaremos a denominar aula A y aula B. La primera está formada por el grupo-clase de cuarto curso de Educación Primaria, integrada por un total de diez alumnos y el propio director del centro, como tutor de la misma, con el que se produjo el primer contacto y se desarrolló una entrevista personal, que proporcionó información general de la participación en el centro y permitió establecer las bases del proceso a seguir. La segunda hace referencia al grupoclase de quinto y sexto, formado por diez alumnos y su tutor, maestro con larga trayectoria en el centro. Además se ha contado con la implicación de familiares del alumnado participante, dando respuesta a un cuestionario individual. Por último, el rol ejercido por la investigadora varía en función de las fases desarrolladas, predominando el papel de observadora en los primeros momentos de la investigación, posteriormente ha tenido lugar una participación más activa, interviniendo en las actividades desarrolladas. 


\subsection{Instrumentos de recogida de información}

Durante el desarrollo del proceso de investigación-acción se han empleado diversas estrategias de recogida de información, favoreciendo la triangulación de los datos, determinadas atendiendo a las características de los participantes y su disponibilidad.

Se han realizado entrevistas individuales semiestructuradas, desarrolladas en el momento inicial, con el objetivo de conocer el nivel de participación del alumnado y las estrategias empleadas al respecto, al director del centro y los tutores de ambas aulas, y en el momento final, a éstos últimos, para conocer la valoración del proceso desarrollado. Dicha estrategia permite obtener información de forma oral y personalizada, partiendo de un guion que determina los aspectos relevantes y permite obtener información rica en matices acerca del primer, segundo y último objetivos de investigación. Con la intención de tener en cuenta la opinión del alumnado, se han organizado grupos de discusión, técnica de carácter colectivo que permite generar un discurso grupal, para conocer percepciones respecto del grado de participación, estrategias empleadas para permitir su escucha, propuestas de acción y su valoración al respecto (Massot, Dorio y Sabariego, 2009). Además, con el objetivo de indagar sobre el grado de implicación del alumnado en la toma de decisiones, las estrategias empleadas y la opinión de los participantes acerca del incremento de la participación, se ha diseñado un cuestionario dirigido al alumnado, respondido individualmente en el aula con ayuda de la investigadora, y otro a las familias, entregado y devuelto por parte del alumnado, con una previa introducción escrita, para conocer la información recibida por las familias acerca de la participación del alumnado. Dicha estrategia consiste en un formulario con preguntas escritas abiertas ( 5 al alumnado y 4 a las familias) y cerradas, con posibilidad de responder "si", "no" o "a veces" (10 al alumnado y 8 a las familias) que permite asegurar una recogida de datos normalizada, al favorecer la espontaneidad y evitar influir en el entrevistado (Alaminos y Castejón, 2015).

De forma paralela, se ha llevado a cabo la observación directa en el interior de las aulas, empleando notas de campo de carácter descriptivo e inferencial como sistema de registro, éstas presentan contenido descriptivo de la situación observada, reflexiones e interpretaciones de la observadora (Massot, Dorio y Sabariego, 2009). Con la finalidad de llevar a cabo el seguimiento del cumplimiento de normas, cuando no es posible la observación directa, y de modo informativo, el alumnado ha empleado un diario de seguimiento, en forma de tabla de doble entrada, en la que constan las normas y la fecha de recogida de la información.

\subsection{Fases de la investigación-acción}

El proceso de investigación-acción se compone de cinco fases, de aspectos generales a concretos, con la finalidad de generar un bucle reflexivo que permita el cumplimiento sucesivo de los objetivos específicos, para alcanzar el objetivo general y dar respuesta a las preguntas de investigación (tabla 1).

\section{Primera fase: Toma de contacto con el centro educativo}

La primera fase consiste en establecer un primer contacto con el centro, mediante una reunión con el director. En ésta se dan a conocer los objetivos de la investigación, compartidos o no por parte del centro en cuestión. Una vez manifestada la conformidad se desarrolla una entrevista con el director, para obtener información relevante y determinar algunas de las bases de la investigación, como las aulas y los participantes. 
Tabla 1. Relación de fases, instrumentos e informantes de la investigación-acción

\begin{tabular}{lll}
\hline \multicolumn{1}{c}{ FASE } & \multicolumn{1}{c}{ INSTRUMENTO } & \multicolumn{1}{c}{ INFORMANTE } \\
\hline Toma de contacto & Encuentro y entrevista & Director \\
\hline & Entrevista semiestructurada & \\
Comprensión de las & Grupo de discusión & Tutores \\
prácticas docentes & Cuestionario & Alumnado \\
& Grabación en audio & Familias \\
& Notas de campo & \\
\hline \multirow{2}{*}{$\begin{array}{c}\text { Gumpartir significados y } \\
\text { orientar la mejora }\end{array}$} & Notas de campo & Tutores \\
& Grabación en audio & Alumnado \\
\hline Puesta en práctica y & Grupo de discusión & Tutores \\
seguimiento del proceso & Notas de campo & Alumnado \\
\hline & Eiario de clase & Tutores \\
Evaluación y reflexión & Grupo de discusión & Alumnado \\
& Grabación en audio &
\end{tabular}

Fuente: Elaboración propia.

\section{Segunda fase: Comprensión de las prácticas docentes. Consulta con el alumnado y las familias}

La segunda fase del proceso de investigación tiene como objetivo recoger información que permita identificar las estrategias puestas en práctica en el contexto del aula, relacionadas con la participación del alumnado. Para ello se emplean diversas estrategias de obtención de datos, permitiendo una comprensión subjetiva y realista.

En primer lugar se desarrolla una entrevista individual a los tutores, para indagar acerca de su perspectiva del alumnado, predisposición a participar, estrategias y metodologías empleadas, etc. Durante la entrevista se pregunta sobre la implicación de las familias en el proceso de enseñanza-aprendizaje, para conocer si se trata de una fuente de información relevante, ante la afirmación de los tutores se desarrolla un cuestionario dirigido a las mismas. En la reunión también se concreta la metodología a emplear para obtener información procedente del alumnado, consensuando el desarrollo de un grupo de discusión en ambas aulas. De forma previa, se pasa un cuestionario individual al alumnado, para establecer un primer contacto y favorecer la participación y escucha de todos en igualdad de condiciones. También se llevan a cabo observaciones, desarrolladas en situaciones escolares diferentes y momentos establecidos por los tutores.

\section{Tercera fase: Compartir significados y orientar la mejora}

Esta fase tiene como finalidad compartir significados y favorecer la toma de decisiones acerca de la propuesta de acción a desarrollar, mediante la devolución de la información a los tutores participantes. Para ello se elabora un breve informe en el que se reflejan aspectos generales de las observaciones, así como las respuestas obtenidas en los cuestionarios al alumnado y las familias. A continuación se desarrolla una reunión conjunta, iniciada con la exposición del proceso a seguir y la entrega del informe correspondiente, una vez leído se produce el intercambio, seguido del diálogo sobre percepciones y propuestas de acción. Ante la situación de indecisión, se realiza una propuesta derivada de la entrevista inicial, consistente en la revisión y establecimiento de nuevas normas de aula por parte del alumnado. Tras la conformidad de los tutores, se desarrolla un grupo de discusión con el alumnado, para recabar información sobre las 
normas de aula, su establecimiento, cumplimiento y conformidad, finalizando con la exposición y aceptación de la propuesta.

\section{Cuarta fase: Puesta en práctica de la propuesta de mejora}

Fase que persigue el objetivo de poner en práctica estrategias que fomenten la participación, la toma de decisiones y la escucha del alumnado dentro del aula. La primera actividad consiste en determinar las normas ya existentes. Crean grupos de trabajo, cada uno encargado de un ámbito sobre el que establecer normas, consecuencias, afectados y quién las hace cumplir. Cada grupo lo expone al resto, la información se recopila en una tabla de doble entrada. Posteriormente se crean grupos encargados de establecer las nuevas normas de cada ámbito, sus consecuencias, el papel del alumnado y del tutor. A medida que se expone cada norma, se debaten y consensuan posibles cambios al respecto, dejándolas por escrito. Dicho proceso tiene una duración de dos sesiones en el aula A y tres en el aula B. Una vez establecidas las nuevas normas se lleva a cabo el seguimiento, mediante un diario de aula desarrollado por los tutores y el alumnado, en el aula A, mientras que en el aula B se decide el establecimiento de un director por día, responsable de favorecer el cumplimiento de normas y consecuencias.

\section{Quinta fase: Evaluación del proceso}

La última fase persigue evaluar las estrategias puestas en práctica, a fin de conocer la opinión de los participantes, identificar posibles beneficios y determinar propuestas de mejora. Se desarrolla la revisión de la documentación recogida al respecto, enriquecida mediante un grupo de discusión con el alumnado de cada aula y una entrevista a los tutores participantes.

\subsection{Análisis de datos}

Una vez registrada la información durante las diferentes fases, se procede a realizar un análisis de datos de carácter cualitativo, mediante el desarrollo de un proceso cíclico, consistente en organizar la información para interpretarla, extraer significados y establecer conclusiones (Rodríguez, 2003). Se procede a identificar los datos relevantes, en relación al objetivo de estudio, y establecer relaciones entre los mismos, por medio de la codificación (González y Cano, 2010). El siguiente paso consiste en la construcción de categorías de carácter mixto, elaboradas tanto de forma deductiva, categorías establecidas a priori, como inductiva, extraídas a partir de la lectura del material recopilado (Rodríguez, 2003). Finaliza el proceso con la exposición de los datos, centrada en organizar y comprender la información ya codificada, por medio de la búsqueda de relaciones entre categorías (González y Cano, 2010).

Durante el proceso de investigación se han tenido en cuenta las normas éticas, respetando el anonimato de los participantes, informándoles de los objetivos de la investigación y lo que sucederá con los datos ofrecidos una vez finalizada, evitando invadir su intimidad, respetando su autonomía y la confidencialidad de los datos obtenidos (Gibbs, 2012). También se ha llevado a cabo el retorno de los datos, concretamente al profesorado, compartiendo y contrastado la información obtenida, para facilitar la interpretación de la información y hacerlos partícipes en el proceso de reflexión de la investigación-acción. 


\section{Resultados}

\section{1. ¿Qué niveles de participación del alumnado se favorecen?}

Centrando la atención en el grado en que se favorece la escucha y expresión de la voz del alumnado, diferenciamos cinco niveles de participación, partiendo de los que le otorgan un mayor protagonismo, hasta llegar a aquellos en los que su implicación se desplaza casi por completo (tabla 2 ).

Tabla 2. Comparación entre los niveles de participación del aula A y aula B

\begin{tabular}{lll}
\hline NivelES DE PARTICIPACIÓN & \multicolumn{1}{c}{ AulA A } & \multicolumn{1}{c}{ AULA B } \\
\hline 1. Escuela democrática & $\begin{array}{c}\text { Desarrollo de actividades, } \\
\text { gestión del aula y } \\
\text { establecimiento de normas }\end{array}$ & - \\
\hline 2. Alumno investigador & Actividades y contenidos & - \\
\hline 3. Alumno participante & $\begin{array}{l}\text { Gestión del aula, organización } \\
\text { de talleres y excursiones, y } \\
\text { establecimiento de } \\
\text { actividades y contenidos }\end{array}$ & Gestión del aula \\
\hline 4. Escucha del alumnado & $\begin{array}{c}\text { Elección de actividades y } \\
\text { contenidos }\end{array}$ & $\begin{array}{c}\text { Gestión del aula, desarrollo de } \\
\text { actividades y contenidos y } \\
\text { organización de talleres y } \\
\text { excursiones }\end{array}$ \\
\hline 5. Ausencia de consulta & Elección de actividades y & $\begin{array}{c}\text { Gestión del aula y elección de } \\
\text { actividades y contenidos }\end{array}$ \\
\hline
\end{tabular}

Fuente: Elaboración propia.

En el aula A se ve reflejado, tanto en el desarrollo de actividades como en la gestión del aula y el establecimiento de normas, un nivel de participación propio de la escuela democrática, debido a la realización de asambleas que favorecen la escucha, discusión y razonamiento de aspectos que preocupan al alumnado, llegando al consenso y provocando cambios en base a las decisiones tomadas. Además las actividades y contenidos se desarrollan de forma predominantemente autónoma por parte del alumnado, el que trabaja a ritmos diferentes en base a un plan de trabajo establecido, aspecto que se relaciona con un segundo nivel de participación, en el que el alumno ocupa el papel de investigador y el tutor asume el rol de acompañante, basado en exponer las actividades y ofrecer las ayudas requeridas por el estudiantado.

El tercer nivel, el que sitúa al alumnado en la posición de participante, predomina en el aula A debido a la frecuente consulta y participación del alumnado, aspecto que según considera el tutor "es muy enriquecedor y se sienten identificados, ya que ven que su voz se tiene en cuenta y se modifican cosas que ellos dicen”. Dicho nivel también se ve reflejado en el desarrollo de ciertas actividades de aula y excursiones, puesto que se escucha al alumnado y se considera su opinión, a pesar de que la decisión final recae con frecuencia sobre el tutor. En referencia al cuarto y quinto nivel, denominados respectivamente escucha del alumnado y ausencia de consulta, se observa una escasa presencia en el aula que nos ocupa. No obstante, ambos se reflejan en las respuestas ofrecidas por el alumnado, al considerar que apenas interviene en la toma de decisiones acerca de los contenidos y ciertas actividades puntuales de aula, como la decisión de realizar una exposición a otros cursos sin contar con la opinión del alumnado. 
Con respecto al aula $\mathrm{B}$ se denota una completa ausencia de los dos primeros niveles de participación, siendo limitada la presencia del tercer nivel, alumno participante, presente en aspectos generales de aula, como el cambio de las persianas tras la petición en un grupo de discusión desarrollado durante la investigación. Se puede constatar el predominio de los dos últimos niveles, referidos a un menor índice de participación. El nivel de escucha del alumnado se caracteriza por la consideración de la totalidad del grupo de que sólo a veces es informado de los cambios producidos, encontrando disparidad de respuestas a la hora de considerar si se pide su opinión tanto en aspectos organizativos como curriculares, únicamente tres alumnos respondieron que sí se realizan actividades para conocer su opinión sobre lo que aprenden. Esto es sostenido por el tutor al afirmar que el alumnado no puede opinar sobre todo, en referencia al tema de organizar excursiones. En relación a las actividades, el alumnado interviene en momentos puntuales, limitando su decisión a opciones ya concretadas"muchas veces el maestro nos ha dicho: ¿os parece bien que demos este tema o os gusta más el otro? Que son temas de pocos días" (alumno 1B).

Por último se observa una clara ausencia de consulta: "antes nos deben pedir nuestra opinión para ver qué nos parece, pero no lo hacen” (alumno 2B), otro alumno manifiesta que recientemente no se les informó de modificaciones en el horario. Por otra parte, el tutor afirma ser él el encargado de preparar las unidades didácticas, refutando en ocasiones las demandas y propuestas del alumnado: "la preparación de las actividades, son unidades didácticas, las preparo yo, se puede decir. Las actividades son bastante libres a la hora de confeccionar". "El alumno no decide cómo va el temario, pero sí que hay proyectos que se van adaptando según como vaya el currículum".

\section{2. ¿Qué mirada mantiene el tutor respecto de la participación del alumnado en su práctica educativa?}

La presente pregunta nos aproxima a conocer el estilo docente que caracteriza a cada maestro, diferenciando entre una mirada renovada, definida como la capacidad para cuestionar su propia práctica y ser flexible ante los cambios, en oposición a una mirada más conservadora, caracterizada por el empleo de metodologías tradicionales y rigidez ante el cambio.

Se puede afirmar que el tutor A presenta una mirada renovada en diversas dimensiones, predominantemente en la gestión del aula, mediante la horizontalidad en la escucha de voces: "dentro del aula somos todos iguales, intento estar al mismo nivel que ellos, es decir, que todas las voces se escuchen por igual. Si tienen algo que decir se escucha, si tiene sentido y estamos de acuerdo pues se hace, y si no se discute". Esto se refleja en la importancia otorgada a la opinión y responsabilidad del alumnado para establecer las normas de aula, proponer y generar cambios, mediante el empleo de dinámicas como la asamblea o el buzón de propuestas, información confirmada por las familias. Tal mirada se mantiene al debatir sobre las actividades, predominando la autonomía por parte del alumnado, respetando los ritmos de aprendizaje, sin sentirse presionado por cumplir todos los contenidos establecidos: "al final de curso los doy a la maestra de tercer ciclo y si hago corto de contenido se lo digo y ya se apaña ella”. En contraposición, el alumnado da a conocer una mirada de carácter conservador a la hora de establecer los contenidos (9 de 10 alumnos responden que no pregunta qué quieren aprender) y otros aspectos, como responsabilizarse íntegramente del cumplimiento de las normas. 
El tutor B se caracteriza por poseer una mirada predominantemente conservadora, como él mismo afirma: "yo soy más tradicional en todo esto" "normalmente llevo yo la voz cantante”. Además la mayoría del alumnado manifiesta que no tiene en cuenta su opinión en la selección de los contenidos. Tampoco ponen en práctica dinámicas de aula estructuradas que favorezcan la escucha, puesto que "el horario está muy restringido y dedicamos tiempo a muchas cosas. En una discusión o marcas muy bien el tiempo o se te puede ir demasiado" y "al estar en tercer ciclo parece que el enfoque sea más directo y quiera ir más a los contenidos" (tutor B). Del mismo modo, las normas de aula las determina el maestro de forma explícita: "no están establecidas, no hay normas porque el alumnado es muy poco y si hay problemas pues se habla en el momento”, encargándose de asegurar su cumplimiento. Dicha mirada se mantiene cuando el tutor afirma que los alumnos no pueden decidir sobre todo, concretamente sobre las excursiones. En contraposición, mantiene una mirada renovada al afirmar: "estamos abiertos a iniciar sistemas de participación”, los alumnos deciden acerca de la organización de talleres, además la mayoría de familiares y alumnado consideran que tiene en cuenta su opinión.

\section{3. ¿Qué estrategias se ponen en práctica para favorecer la voz del alumnado?}

Las estrategias empleadas para favorecer la escucha del alumnado en el centro educativo en general, como indica el director, se centran en la preparación de su voz en cada aula, así como el establecimiento de un portavoz encargado de darla a conocer en la asamblea desarrollada por la Comisión Coordinadora. Además, el tutor B y el alumnado, mencionan la realización de asambleas, charlas, formularios de consulta y dinámicas como "los sueños" y "el buzón”, para conocer lo que cada uno desea u opina de la escuela. No obstante, esta voz se pierde con facilidad debido al poco tiempo dedicado a las dinámicas de preparación, por lo que el tutor B propone el establecimiento sistemático de asambleas de aula, aulario y centro.

Concretando en las aulas participantes, en el aula A se desarrollan dos estrategias estructuradas que favorecen la escucha. Por una parte, se pone en práctica la técnica “propongo, felicito y pido perdón”, consistente en que el alumnado escriba su opinión y la introduzca en el buzón del aula. Dicha dinámica deriva en el desarrollo de una asamblea quincenal, en la que abren el buzón y leen su contenido, con la finalidad de comentar y dar solución a aquellos aspectos que preocupan al alumnado. En este sentido, el tutor plantea que "el problema para esas estrategias es la falta de tiempo, yo dedico la sesión asamblearia quincenal, que no siempre cae cuando hace falta, entonces les digo: apuntarlas y el lunes lo hablamos en la asamblea, aunque no toque", además manifiesta "eso me ha ido bien una temporada, este último año no ha funcionado tanto porque al ser diez hay poco conflicto y no hay papeles". Por otra parte, trimestralmente tiene lugar una asamblea en la que participan familiares, alumnado y tutor, el que previamente ayuda a preparar la voz al alumnado, respecto a aspectos positivos, negativos y propuestas de mejora de lo sucedido en el aula. No obstante, el alumnado propone desarrollar más reuniones junto con los maestros y las familias.

En referencia al aula $\mathrm{B}$, las estrategias no se plantean estructuradamente, puesto que como afirma el tutor: "no hay una asignación de asamblea cada día, sino que se va resolviendo y se habla”. Además hace referencia a la dificultad presente para establecer estrategias concretas que permitan lograr que la voz del alumnado sea real, ante la que propone el establecimiento de asambleas de forma sistemáticas como un paso importante. La mayoría de las familias desconocen las estrategias y el alumnado reclama 
una mayor escucha individualizada por parte de los maestros, empleando el lenguaje oral.

\section{4. ¿Qué estrategias establecer para favorecer la expresión y escucha del alumnado?}

A pesar de la existencia de gran variedad de estrategias para mejorar la participación del alumnado, la investigación conduce a centrarse en el establecimiento de normas de aula por parte del alumnado. Previamente a la intervención, en el aula A se concretó un decálogo de normas a principio de curso, de forma conjunta entre el alumnado y el tutor, a diferencia del aula $\mathrm{B}$, en la que no estaban establecidas, pero eran determinas por el tutor, encargado de asegurar su cumplimiento en ambas aulas. Durante el establecimiento, las dos aulas coinciden al proponer normas similares a las ya existentes, no obstante encontramos una clara diferencia al determinar el responsable del cumplimiento. En el aula A el alumnado decide que su papel será recordar las normas, al igual que el del tutor, a quien además responsabilizan de reñir y castigar, dependiendo de la consecuencia. En el aula B se toma la decisión de establecer la figura del "director de las normas", alumno responsable de controlar el cumplimiento y avisar al tutor de las consecuencias pertinentes al final del día, a quien otorgan el papel de hacer cumplir las consecuencias, dar el turno de palabra, reñir y enviar una nota a los padres.

El cumplimiento de las normas en general ha sido satisfactorio, al igual que el desempeño de roles establecidos, a pesar de que el papel del tutor continua siendo relevante. El alumnado destaca como aspectos positivos el incremento del orden y la convivencia en las aulas, así como el aumento de su protagonismo y responsabilidad. Además el tutor A manifiesta que ha permitido "que sean conscientes de que las normas no están ahí porque sí, sino que están porque en la convivencia con mucha gente hacen falta muchas normas para estar todos juntos, sino sería imposible", a lo que cabe añadir la positiva valoración del mismo acerca de determinar quién controla las normas, y realizar su valoración y seguimiento. Con respecto a los aspectos negativos, destaca que algunos alumnos del aula B no consideran necesario el establecimiento de normas, afirmando que puede causar conflictos entre ellos y que el control de su cumplimiento interfiere en la práctica educativa. Por último, el tutor $\mathrm{B}$ opina que ha habido falta de consenso en el establecimiento, al afirmar que "no es fácil, porque a veces pensamos que es una norma de todo el grupo, pero es una norma del líder” y hace referencia a la falta de tiempo de intervención para observar resultados.

Partiendo de lo ya mencionado, las propuestas de mejora planteadas por el alumnado del aula B se centran en realizar cambios en el papel del director, como ser responsable y justo, estableciendo un nuevo director en caso contrario. El alumnado de ambas aulas propone "ponerlas en la pared para recordarlas, sobre todo las que menos cumplimos, como levantar la mano para hablar". Por su parte, los tutores manifiestan la necesidad de desarrollar la estrategia desde principio de curso y de forma más pausada. Para finalizar, tanto el alumnado como los tutores manifiestan el deseo de repetir el proceso desarrollado en el próximo curso escolar. 


\section{Conclusiones y discusión}

El desarrollo de la investigación nos ha permitido aprender ciertos aspectos sobre la participación del alumnado y las estrategias empleadas para favorecerla, así como conocer la interrelación existente entre los objetivos planteados y abrir nuevos interrogantes, que a continuación se expone a modo de conclusiones.

En primer lugar, el objetivo de indagar sobre los ámbitos y niveles de participación del alumnado en el centro educativo y el aula, que da respuesta a la primera pregunta de investigación, permite afirmar que en el aula A están presentes los cinco niveles de participación anteriormente descritos. No obstante, predomina el nivel denominado alumno participante, comprometido con una participación real del alumnado, caracterizada por el interés por conocer su opinión, propiciando un mayor debate y su autonomía, otorgando relevancia a la voz del alumnado para gestionar el cambio (Susinos y Ceballos, 2012). En el aula B predomina el nivel de escucha del alumnado, puesto que éste representa una mera fuente de datos, siendo el profesorado el encargado de procesar la información obtenida y actuar en consecuencia (Nieto y Portela, 2008). En este sentido, encontramos una clara interrelación entre los niveles de participación adquiridos en cada aula y la mirada que el tutor otorga a su práctica docente, pudiendo confirmar que para avanzar hacia niveles en los que la voz del alumnado adquiere un mayor protagonismo "es imprescindible que los profesores puedan analizar sus propias prácticas con una mirada renovada, desde un pensamiento más amplio, complejo y comprometido sobre las posibilidades de la participación de los estudiantes en la mejora de la vida escolar" (Susinos y Ceballos, 2012, p. 40).

En segundo lugar, el objetivo de identificar las estrategias empleadas para permitir la participación y tener en cuenta la voz el alumnado en el contexto del aula, da respuesta a la segunda pregunta de investigación, afirmando que en el aula A se desarrollan estrategias que favorecen la escucha del alumnado, estructuradas y desarrolladas de forma sistemática, mientras que en el aula $\mathrm{B}$ no se pone en práctica ninguna metodología concreta. Tal afirmación nos remite al párrafo anterior, pudiendo establecer relación entre el nivel de participación, la mirada del tutor y las estrategias desarrolladas, puesto que el nivel de participación más elevado, denominado por Fielding (2011) "escuela democrática", presente en el aula A y ausente en la B, contempla la faceta de que "la escuela opere alguna forma de 'responsabilidad compartida' en la cual la planilla y los alumnos se reúnan regularmente como comunidad/sub-comunidad (diaria, semanal o trimestralmente) para reflexionar sobre su trabajo, compartir sus aspiraciones y decidir cursos de acción apropiados" (p. 39).

Por último, los objetivos centrados en compartir significados sobre la participación y favorecer la toma de decisiones compartida, y poner en práctica y evaluar estrategias que fomenten la participación, la toma de decisiones y la escucha del alumnado dentro del aula, nos llevan a establecer la estrategia de involucrar al alumnado en la reelaboración de las normas del aula, mediante la que se les ofrece la oportunidad de construir su ciudadanía democrática, ejerciendo sus derechos y aprendiendo a respetar sus deberes (Le Gal, 2005). Poner en práctica dicha metodología ha permitido que el alumnado en general sienta que se le considera agente activo y se le tiene en cuenta, incrementando su responsabilidad y avanzando hacia una autonomía gradual (San Fabián, 2008), lo que ha requerido la presencia e influencia del tutor, al que el grupo ya atribuye la posición de gestionador de la autoridad (Lorenzo, 2004). En este sentido, cabe hacer referencia a la 
importancia atribuida al establecimiento de la forma de gestionar el cumplimiento de las normas, papel que resulta relevante otorgar al propio alumnado, aspecto que se puede llevar a cabo mediante el reparto de cargos, estableciendo unas bases consensuadas para favorecer que tal decisión no implique conflictos en las relaciones y/o interferencias en la práctica educativas.

En este sentido, resulta conveniente trabajar de forma gradual el establecimiento de cada una de las normas, con la intención de determinar las consecuencias apropiadas y establecer los papeles a ejercer, teniendo en cuenta el nivel de cumplimiento de cada una de ellas y el grado de responsabilidad que es capaz de asumir el alumnado, favoreciendo una correcta asimilación. Dicho proceso no se ha podido considerar durante el desarrollo de la investigación debido a la escasez de tiempo de intervención con el que se ha contado, como consecuencia de haber sido llevada a cabo en las últimas semanas del curso escolar, a diferencia de las recomendaciones de Pérez (1995): "el período de tiempo en el que se debe planificar el aprendizaje de las normas básicas debe ser medio o largo (un ciclo o una etapa educativa), al objeto de que permanezcan lo más estables posibles y se facilite su asimilación” (p. 6).

A pesar de tales limitaciones, se ha podido alcanzar el incremento de la participación del alumnado en ambas aulas. Además los propios participantes han podido tomar conciencia de los aspectos positivos que se asocian a la estrategia, entre los que cabe destacar el aumento del orden, la convivencia y la responsabilidad (San Fabián, 2008). Ello invita a reflexionar sobre la posibilidad de implantar estrategias que otorguen mayor protagonismo al alumnado y favorezcan la escucha de su voz, aun teniendo en cuenta la estrecha relación existente entre la mirada que otorga el maestro a su práctica docente, las diferentes estrategias participativas empleadas y los niveles de participación presentes en las aulas. Todo ello abre camino a nuevas vías de investigación, centradas en comprobar la influencia que ejerce sobre la mirada del docente acerca de la voz del alumnado, el hecho de poder experimentar de forma directa los beneficios proporcionados por estrategias que favorecen avanzar hacia un nivel de participación propio de la escuela democrática (Fielding, 2011).

Para finalizar, cabe hacer referencia a los beneficios aportados mediante el desarrollo de la investigación-acción, la que ha permitido que el alumnado tenga la oportunidad de incrementar su protagonismo y sentirse partícipe de un proceso de transformación que le implica de forma directa. Por otra parte, involucrar a maestros en la investigación ha favorecido el aumento de interés acerca de la importancia y posibilidad de tener en cuenta la voz del alumnado, mediante el establecimiento de estrategias concretas, aspecto que permite encauzar reflexiones críticas sobre su práctica educativa desde un enfoque más intercultural e inclusivo.

\section{Referencias}

Alaminos, A. y Castejón, J. L. (2015). Elaboración, análisis e interpretación de encuestas, cuestionarios y escalas de opinión. Valencia: Marfil.

Elliott, J. (2011). Educational action research and the teacher. Recuperado de http://www.quadernsdigitals.net/datos/hemeroteca/r_75/nr_824/a_11127/11127.html 
Fielding, M. (2011). La voz del alumnado y la inclusión educativa: una aproximación democrática radical para el aprendizaje intergeneracional. Revista Interuniversitaria de Formación del Profesorado, 7O(1), 31-61.

Gibbs, G. (2012). El análisis de datos cualitativos en investigación cualitativa. Madrid: Morata.

González, T. y Cano, A. (2010). Introducción al análisis de datos en investigación cualitativa: tipos de análisis y proceso de codificación. NURE Investigación, 45, 1-10.

Latorre, A. (2003). La investigación acción. Conocer y cambiar la práctica educativa. Barcelona: Graó.

Le Gal, J. (2005). Los derechos del niño en la escuela. Una educación para la ciudadanía. Barcelona: Graó.

Lorenzo, M. (2004). Conflictos, tutoría y construcción democrática de las normas. Bilbao: Desclée De Brouwer.

Martínez, J. (2006). La participación de los alumnos en los centros. Sentido y realidad. Participación educativa. Revista Cuatrimestral del Consejo Escolar del Estado, 2, 37-43.

Massot, I., Dorio, I. y Sabariego, M. (2009). Estrategias de recogida y análisis de la información. En R. Bisquerra (Ed.), Metodología de la investigación educativa (pp. 329-365). Madrid: La Muralla.

Moliner, O. y St-Vicent, L. A. (2014). Dilemas éticos de los investigadores que acompañan procesos de investigación-acción, en el marco de la escuela intercultural inclusiva. Revista Latinoamericana de Educación Inclusiva, 8(2), 49-68.

Montalbán, R. (2006). La participación estudiantil. Participación Educativa, 2, 33-36.

Nieto, J. y Portela, A. (2008). La inclusión de la voz del alumno en el asesoramiento para la mejora de las prácticas educativas. Profesorado, Revista de Currículum y Formación de Profesorado, 12(1), 1-26.

Pérez, C. (1995). El aprendizaje de normas en el aula mediante la participación democrática. Revista Aula de Innovación Educativa, 45, 1-24.

Pérez, C. (2007). Efectos de la aplicación de un programa de educación para la convivencia sobre el clima social del aula en un curso de $2^{\circ}$ de ESO. Revista de Educación, 343, 503-529.

Pérez, C. (octubre, 2011). Autonomía y responsabilidad como valores clave para la elaboración de normas de convivencia en la ESO. Comunicación presentada en el XII Congreso Internacional de Teoría de la Educación. Universitat de Barcelona.

Ramos, S. (2013). Son cosas de niños. La participación como derecho y la educación inclusiva: reflexión en torno al papel de las niñas y niños en la escuela. Revista Latinoamericana de Educación Inclusiva, 7(1), 151-167.

Rodríguez, C. (febrero, 2003). Nociones y destrezas básicas sobre el análisis de datos cualitativos. Comunicación presentada en el Seminario internacional: el proceso de investigación en educación, algunos elementos clave. Santo Domingo.

Rojas, S., Haya, I. y Lázaro-Visa, S. (2012). La voz del alumnado en la mejora escolar: niños y niñas como investigadores en educación primaria. Revista de Educación, 359, 81-101. doi:10.4438/1988-592X-RE-2012-359-197

Rudduck, J. y Flutter, J. (2007). Cómo mejorar tu centro escolar dando voz al alumnado. Madrid: Morata.

Sales, A., Moliner, O. y Ruiz, P. (2013) Procesos de transformación hacia la escuela intercultural inclusiva desde la gestión participativa. En M. Navarro y A. Barraza (Eds.), Dirección, 
liderazgo y modelos y procesos de gestión: claves hacia la transformación (pp. 248-282). México: Red Durango de Investigadores Educativos, AC.

Sales, A. (2011). Creando redes para una ciudadanía crítica desde la escuela intercultural inclusiva. Revista Educación Inclusiva, 5(1), 51-68.

San Fabián, J. (2008). La voz de los estudiantes en los centros escolares. ¿Hay alguien ahí? Organización y Gestión Educativa: Revista del Fórum Europeo de Administradores de la Educación, 16(5), 27-32.

Susinos, T. (2012). Presentación. Las posibilidades de la voz del alumnado para el cambio y la mejora educativa. Revista de Educación, 359, 16-23.

Susinos, T. y Ceballos, N. (2012). Voz del alumnado y presencia participativa en la vida escolar. Apuntes para una cartografía de la voz del alumnado en la mejora educativa. Revista de Educación, 259, 24-44. doi: 10.4438/1988-592X-RE-2012-359-194

Susinos, T. y Rodríguez-Hoyos, C. (2011). La educación inclusiva hoy. Reconocer al otro y crear comunidad a través del diálogo y la participación. Revista Interuniversitaria de Formación del Profesorado, 70(25), 15-30.

Susinos, T., Rojas, S. y Lázaro, S. (2011). La voz del alumnado y el cambio escolar en el camino hacia la inclusión educativa: aprendiendo de la experiencia de un centro de educación infantil y primaria. Revista Latinoamericana de Educación Inclusiva, 5(2), 83-99.

Traver, J.A., Sales, A., Moliner, O. (2010). Ampliando Territorio: Algunas Claves sobre la Participación de la Comunidad Educativa. REICE. Revista Iberoamericana sobre Calidad, Eficacia y Cambio en Educación 8(3), 97-119.

\section{Breve CV de las autoras}

\section{Marta Terrén}

Graduada en Maestra de Educación Infantil y Máster Universitario en Psicopedagogía, especialidad en Intervención Psicopedagógica desde la Mejora Educativa y la Inclusión, en la Universidad Jaume I. Colaboradora en el grupo de investigación MEICRI (Mejora Educativa y Ciudadania Crítica) en proyecto de investigación y cursos de formación del profesorado, así como en talleres de habilidades cooperativas dirigidas al alumnado universitario. Email: al202175@uji.es

\section{Auxiliadora Sales}

Doctora en Ciencias de la Educación y Profesora Titular del Departamento de Educación, Área de Didáctica y Organización Escolar, de la Universitat Jaume I, en Castellón. Ha centrado su investigación en educación intercultural inclusiva, formación del profesorado, investigación-acción y cambio escolar. Miembro del grupo de investigación MEICRI (Mejora Educativa y Ciudadania Crítica), con el que ha desarrollado proyectos de investigación sobre la construcción de la escuela intercultural inclusiva y de participación comunitaria y ciudadana para una escuela incluida en el territorio.Email: asales@uji.es 